\title{
Biomaterials
}

\section{The role of trabecular demineralized bone in combination with perichondrium in the generation of cartilage grafts}

\author{
Gerjo J.V.M. van Osch ${ }^{\mathrm{a}, *}$, Paul G.J. ten Koppel ${ }^{\mathrm{a}}$, Simone W. van der Veen ${ }^{\mathrm{a}}$, Pawel Poppe ${ }^{\mathrm{a}, \mathrm{b}}$, \\ Elisabeth H. Burger ${ }^{c}$, Henriette L. Verwoerd-Verhoef ${ }^{a}$ \\ ${ }^{a}$ Department of Otorhinolaryngology, Head and Neck Surgery, University Hospital, Rotterdam, The Netherlands \\ ${ }^{\mathrm{b}}$ Department of Pathophysiology and Clinic of Otorhinolaryngology, CMKP, Warsaw, Poland \\ ${ }^{\mathrm{c}}$ Department of Oral Cell Biology, Academic Center for Dentistry Amsterdam, Free University Amsterdam, The Netherlands
}

Received 26 February 1998; accepted 12 July 1998

\begin{abstract}
The use of a composite graft of bovine trabecular demineralized bone matrix (DBM) and perichondrium has been found a reliable method for in vivo generation of cartilage. In the present study, the mechanism whereby this commercially available matrix increases cartilage formation was investigated.

First, the time course of cartilage formation in vivo, in the combined implant of perichondrium and DBM in the rabbit ear was studied, with special focus on tissue reactions to DBM. DBM was colonized by macrophages from day 3 post-operatively, reaching a maximum after 2 weeks. Only a minimal number of neutrophils was found. After 3 weeks the DBM appeared to be resorbed. In the first week the DBM was invaded with chondroblasts, and chondrogenesis occurred between the first and second week of implantation. After 3 weeks, the initially formed islets of cartilage had fused.

Next, the chondrogenic capacity of DBM itself was investigated by implantation of DBM without perichondrium. This never resulted in cartilage formation. Immunohistochemistry showed only a faint staining of the DBM for growth factors. This indicates a minimal chondrogenic effect of DBM alone and the requirement of perichondrium as cell provider.

In order to define the conditions which cause chondrogenesis in composites of perichondrium and DBM, a series of in vitro culture experiments was performed in which the in vivo situation was mimicked step by step. The basic condition was perichondrium cultured in medium with $10 \%$ FCS. In this condition, cartilage formation was variable. Because in the in vivo situation both DBM and macrophages can release growth factors, the effect of IGF1, TGF $\beta 2$ or OP1 added to the culture medium was tested. Neither the incidence nor the amount of cartilage formation was stimulated by addition of growth factors. Perichondrium wrapped around DBM in vitro gave cartilage formation in the perichondrium but the incidence and amount were not significantly stimulated compared to cultures of perichondrium without DBM. However, cartilage-like cells were found in the DBM suggesting an effect of DBM on perichondrium-derived cells. Finally, macrophages and/or blood were added to the composite DBM-perichondrium to mimic the in vivo situation as close as possible. However, no effect of this treatment was found.

In conclusion, this study indicates that DBM itself has few chondrogenic qualities but functions merely as a spacer for cell ingrowth. The fast resorption of DBM by macrophages in vivo seems of importance for the cartilage forming process, but in vitro the presence of macrophages (in combination with blood) could not enhance chondrogenesis. (C) 1999 Elsevier Science Ltd. All rights reserved
\end{abstract}

Keywords: Demineralized bone matrix; Perichondrium; Chondrogenesis; Growth factors; Macrophages

\section{Introduction}

Defects in the cartilage of nose, cricoid, ear or articular joints show limited tendency of spontaneous healing. For repair of these defects with transplants, the use of

*Corresponding author. Tel.: +3110 4087661; fax: +31 104367146 . autologous tissue is preferred in order to avoid immunological reactions and the risk of transmittable diseases. The amount of autologous cartilage that can be harvested is limited and the consequent donor site morbidity is high. In contrast, isolation of autologous perichondrium is relatively easy for patient and surgeon and results in minimal donor site morbidity and a low complication rate. Therefore, perichondrium has been 
suggested as a producer of new cartilage [1-5]. The amount of cartilage generated from perichondrium in vivo, however, appeared to be variable and unpredictable [1-3]. In previous research, our group has demonstrated a new experimental method using rabbits: a composite graft of trabecular demineralized bone matrix (DBM) and perichondrium can provide much more consistent results $[6,7]$. Perichondrium wrapped around DBM leads to gradual replacement of the DBM by autologous cartilage tissue [6] even in the child [8]. Implanted subcutaneously in the ear or intramuscularly in the quadriceps of rabbits the grafts showed cartilage formation in $100 \%$ of the samples after 3-6 weeks [7].

In our studies a trabecular matrix (DBM, Osteovit ${ }^{\mathbb{R}}$ ) was chosen because the porosity of the matrix was considered of importance for the ingrowth of cells. The role of DBM for chondrogenesis could be 'physical', by acting as a spacer for cell ingrowth or 'chemical', induced by growth factors which are suggested to be present in the matrix. The aim of this study was to investigate the role of the trabecular DBM in this process in more detail. We first studied the time course of the cartilage generating process in vivo with special focus on cellular responses to DBM. The chondrogenic capacities of the material itself were evaluated by implantation of the material without perichondrium. In the literature, cortical DBM is often described to induce chondro- and osteogenesis and is demonstrated to contain growth factors $[9,10]$. We studied the presence of growth factors in our matrix using immunohistochemistry. Since TGF $\beta$, BMP and IGF are recognized as factors present in DBM $[9,10]$ and are described to induce chondrogenesis [11-15], they were added to perichondrium in culture to study the effects on chondrogenesis. In a further attempt to define the conditions that permit chondrogenesis in the composite of perichondrium and DBM, we mimicked the in vivo procedure in culture. Perichondrium was wrapped around DBM and cultured in vitro and the effects of addition of macrophages and blood was evaluated on histology.

\section{Materials and methods}

The various experimental conditions are outlined in Table 1.

\subsection{In vivo studies}

Twelve female New Zealand White rabbits were used (weighing 1200-1800 g, age 6-12 weeks). Anaesthesia was given with xylazine-hydrochloride (Rompun, Bayer, Leverkusen, Germany) $10 \mathrm{mg} \mathrm{kg}^{-1}$ body weight and ketamine-hydrochloride (Ketalin, Apharma, Arnhem, Netherlands) $50 \mathrm{mg} \mathrm{kg}^{-1}$ body weight via intramuscular injection.
Table 1

Overview of all experimental conditions tested in vivo and in vitro with time of harvesting (time) and sample size (n)

\begin{tabular}{|c|c|c|}
\hline Experimental conditions & Time & $n$ \\
\hline \multicolumn{3}{|l|}{ (a) In vivo experiments with $D B M$} \\
\hline \multirow[t]{4}{*}{ DBM with perichondrium } & $\mathrm{d} 3$ & 6 \\
\hline & wk1 & 6 \\
\hline & wk2 & 6 \\
\hline & wk3 & 6 \\
\hline \multirow[t]{3}{*}{ DBM without perichondrium } & wk1 & 6 \\
\hline & wk2 & 6 \\
\hline & wk3 & 6 \\
\hline \multicolumn{3}{|c|}{ (b) In vitro experiments of perichondrium with growth factors } \\
\hline Perichondrium + FCS & wk3 & 66 \\
\hline Perichondrium $+\mathrm{FCS}+\mathrm{TGF} \beta$ & wk3 & 17 \\
\hline Perichondrium + FCS + OP1 & wk3 & 16 \\
\hline Perichondrium + FCS + IGF1 & wk3 & 15 \\
\hline Perichondrium $-\mathrm{FCS}+\mathrm{IGF} 1+\mathrm{TGF} \beta 2$ & wk3 & 15 \\
\hline \multicolumn{3}{|c|}{$\begin{array}{l}\text { (c) In vitro experiments of perichondrium with DBM, cultured } \\
\text { in } 10 \% \text { FCS }\end{array}$} \\
\hline Perichondrium - DBM & wk3 & 66 \\
\hline Perichondrium + DBM & wk3 & 47 \\
\hline Perichondrium + DBM + macrophages & wk3 & 10 \\
\hline Perichondrium + DBM + blood & wk3 & 14 \\
\hline Perichondrium + DBM + macrophages + blood & wk3 & 10 \\
\hline Perichondrium + DBM colonized in vivo & wk3 & 5 \\
\hline DBM colonized in vivo & wk3 & 5 \\
\hline
\end{tabular}

The operation site was shaved and disinfected with $70 \%$ ethanol. After making a rectangular incision (approximately $2 \times 6 \mathrm{~cm}^{2}$ ) at the concave side of the ear, the skin was elevated and the perichondrium dissected from the underlying cartilage. A rectangular piece of trabecular DBM (Osteovit ${ }^{\mathbb{R}}$, Braun Gmbh, Melsungen, Germany) measuring approximately $4 \times 4 \times 8 \mathrm{~mm}^{3}$ was soaked in blood. This block was wrapped in perichondrium such that the perichondrial side dissected off the cartilage (the cambium layer) faced the DBM surface, and left in situ in the ear for 3 days, 1, 2 or 3 weeks. Furthermore, DBM was implanted subcutaneously without perichondrial envelope and left in the ear for 1,3 or 6 weeks.

\subsection{In vitro studies}

Ear perichondrium was obtained from 15 young female New Zealand White rabbits (age 6-12 weeks) as described above. After washing with physiological saline containing gentamicin $\left(50 \mu \mathrm{g} \mathrm{ml}^{-1}\right)$ and fungizone $\left(0.5 \mu \mathrm{g} \mathrm{ml}^{-1}\right)$ to remove blood and contaminants, the perichondrium was cut into pieces of about $2 \mathrm{~mm}^{2}$ using scalpels. Four explants were cultured per well of a 24wells plate in DMEM/Ham's F12 $(1: 1)$ medium (Life Technologies, Breda, The Netherlands) containing 10\% heat-inactivated FCS, $25 \mu \mathrm{g} \mathrm{ml}^{-1}$ L-ascorbic acid (Sigma), $50 \mu \mathrm{g} \mathrm{ml}^{-1}$ gentamicin and $0.5 \mu \mathrm{g} \mathrm{ml}^{-1}$ fungizone. The 
explants were cultured for 3 weeks and medium was changed three times per week. Results were evaluated using histological analysis.

The in vitro studies attempted to mimic step by step the in vivo situation (Fig. 1). As possible chondrogenic factors, growth factors were added to cultures of perichondrium or perichondrium was combined with DBM. Addition of macrophages, important for resorption of the matrix in-vivo, was studied next. Finally, DBM was 'colonized' by cells in vivo before it was enwrapped with perichondrium and subsequently cultured in vitro.

To test the effects of growth factors, rhTGF- $\beta 2$ (Sandoz, Switzerland), rhOP-1 (Creative Biomolecules, Hopkinton, Massachusetts) or rhIGF-1 (BoehringerMannheim, Almere, The Netherlands) were added to ear perichondrium cultured in medium with $10 \%$ FCS. TGF- $\beta 2$ and OP- 1 were added in a concentration of $10 \mathrm{ng} \mathrm{ml}^{-1}$ continuously or as a pulse treatment in a concentration of $100 \mathrm{ng} \mathrm{ml}^{-1}$ for the first 2 days only. IGF-1 was added in a concentration of $10 \mathrm{ng} \mathrm{ml}^{-1}$ continuously. Furthermore, the effect of a combination of $10 \mathrm{ng} \mathrm{ml}^{-1}$ IGF- 1 and $10 \mathrm{ng} \mathrm{ml}^{-1}$ TGF- $\beta 2$ added for 3 weeks was tested under serum-free conditions in DMEM/Ham's F12 medium with $0.1 \%$ BSA and $25 \mu \mathrm{g} \mathrm{ml}^{-1}$ L-ascorbic acid. All concentrations were based on data from literature [11-17].

DBM was cut into pieces of $2-6 \mathrm{~mm}^{3}$ and a combination of DBM and perichondrium were tested under the following conditions:

(1) Perichondrium explants excised at a size of approximately $2 \times 7 \mathrm{~mm}$ and wrapped around the DBM matrix, with the layer which faced the cartilage towards the DBM, and cultured for 3 weeks.

(2) DBM seeded with macrophages before perichondrium was wrapped around it. Autologous macrophages were isolated together with the perichondrium by washing the peritoneal cavity of the rabbit with PBS immediately after killing the animal. The peritoneal cavity was opened with a small incision and 0.3-0.5 1 PBS was introduced in the cavity. After closing the incision, the abdomen was gently massaged to allow solvation of peritoneal macrophages in PBS. Then, as much PBS (with cells) as possible was retained from the cavity through a disposable needle $(1.1 \mathrm{~mm} \times 40 \mathrm{~mm})$. The cells were separated from the solution by centrifugation. Cytospins stained with $\mathrm{mAb}$ CD68 showed that $>95 \%$ of the isolated cells were macrophages. After washing, the cells were seeded in DBM in a density of $5 \times 10^{7}$ cells $\mathrm{ml}^{-1}$ to allow a large amount of macrophages to adhere to and degrade the DBM. After incubation for at least $30 \mathrm{~min}$, the DBM-macrophage composite was enwrapped in perichondrium.

(3) DBM (with or without macrophages) soaked in autologous blood and cultured in vitro.
A

isolated perichondrium

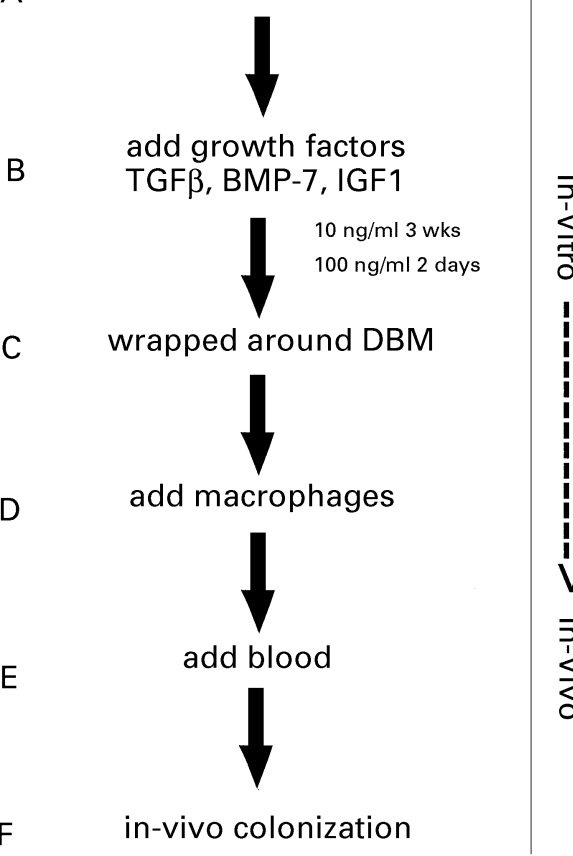

Fig. 1. Experimental design of in vitro conditions to mimic step by step the in vivo situation.

(4) DBM pre-incubated in vivo by implantation of $10 \times 10 \times 3 \mathrm{~mm}$ DBM subcutaneously in the ear for 6 days. After harvesting, the DBM was cultured with or without perichondrial envelope.

\subsection{Histology}

\subsubsection{Presence of growth factors in DBM}

Cryosections of pure DBM were prepared. Sections of human demineralized bone were used as control. To evaluate the effectiveness of the demineralization procedure, staining according to Goldner was used. Immunohistochemical staining for growth factors IGF1 (using a rabbit polyclonal, GroPep, Adelaide, Australia), TGF $\beta 2$ and 3 (using rabbit polyclonals 1: 50, Santa Cruz Biotechnology, CA) was performed after formalin fixation in the absence and presence of saponin. A signal was made visible using 3-amino-9-ethylcarbazole (AEC) as substrate.

\subsubsection{In vivo cartilage formation}

To study the chondrogenic potential of DBM, implants without perichondrium were harvested after 1, 3 or 6 weeks. Implants with perichondrium were harvested after 3 days, 1,2 and 3 weeks $(n=6$ for each time point) to assess chondrogenesis and cellular response to DBM. All specimens were cut into two equal parts; one part was fixed in $4 \%$ phosphate-buffered formalin, decalcified with $10 \%$ EDTA and embedded in paraffin. Serial sections were cut $(7 \mu \mathrm{m})$ and stained with Alcian Blue 8GX (Sigma, St Louis, MO) to study cartilage matrix 
formation. The other part was frozen in liquid nitrogen and stored at $-80^{\circ} \mathrm{C}$ until cryosections $(6 \mu \mathrm{m})$ were made. Immunohistochemistry was performed on these sections to characterize the matrix generated and to evaluate the cell-mediated resorption of DBM. Sections were fixed in acetone. For collagen staining the sections were treated with hyaluronidase (Sigma, St Louis, MO), for chondroitin sulfate staining the sections were treated with chondroitinase ABC (Sigma). The sections were incubated overnight at $4^{\circ} \mathrm{C}$ with $\mathrm{mAb}$ against collagen type II (CIICI; 1: 100; Developmental Studies Hybridoma Bank), pro-collagen type I (M38; 1:1000; Developmental Studies Hybridoma Bank) or chondroitin 6-sulfate (3B3; 1:1000; ICN Biomedicals, Costa Mesa, CA). To study host tissue reactions against DBM, sections were incubated for $2 \mathrm{~h}$ at room temperature with mAbs against macrophages using CD68 (1:50; Behring, Marburg, Germany) and neutrophils using $\alpha$-lactoferrine (1:25; Pharmingen, San Diego, CA). After incubation with primary antibodies, sections were treated with goat-anti-mouse biotin, followed by streptavidin alkaline phosphatase (Supersensitive, Biogenics, Clinipath, Duiven, The Netherlands). Alkaline phosphatase activity was demonstrated using a New Fuchsin substrate (Chroma, Kongen, Germany). This resulted in a red coloured signal. Endogenous alkaline phosphatase activity was inhibited with levamisol (Sigma). Sections were counterstained with Gill's haematoxylin and embedded in gelatin-glycerin.

\subsubsection{In vitro cartilage formation}

After three weeks in vitro, the explants were harvested, fixed in $4 \%$ phosphate buffered formalin and embedded in paraffin. Sections of $6 \mu \mathrm{m}$ thickness were cut and four sections, spaced $60 \mu \mathrm{m}$ apart, were mounted on one slide, stained with Alcian Blue 8GX and counterstained with Nuclear Fast Red.

The incidence of new cartilage formation in the explants was scored. A Fisher's exact test was used for statistical analysis. The percentage of the area stained with Alcian Blue was quantified using image analysis using Videoplan software (Kontron, Zeiss, The Netherlands) using two high quality sections at least $200 \mu \mathrm{m}$ apart, for each sample. Since small pieces of cartilage could be included when perichondrium was dissected (accounting for up to $10 \%$ of the tissue area), distinction was made between pre-existing and newly formed cartilage based on the intensity of Alcian Blue staining and the morphology of the cells. The amount of newly formed cartilage was expressed as a percentage of the total area corrected for pre-existing cartilage and calculated as follows:

Area newly formed cartilage/

(Total area - Area pre-existing cartilage).
The median and range were calculated and Kruskall Wallis one-way ANOVA and rank sum test were used for statistical analysis. A $P$ value $<0.05$ was considered statistically significant.

Finally, immunohistochemical stainings for collagen type II was performed on the paraffin sections. Sections were pretreated with pronase type XIV (Sigma) to regain antigenicity which was lost due to formalin fixation, and with hyaluronidase (Sigma) to obtain better antibody penetration. Incubation with monoclonal antibody IIII6B3 (Developmental Studies Hybridoma Bank) was followed by incubation with a second antibody conjugated with alkaline phosphatase. Alkaline phosphatase activity was demonstrated with a New Fuchsin substrate, resulting in a red color.

\section{Results}

\subsection{In vivo}

\subsubsection{DBM without perichondrium}

Without perichondrial cover, no cartilage or bone was ever formed subcutaneously in the DBM. After three weeks, the DBM was completely resorbed in three out of six samples. After 6 weeks complete resorption of DBM had occurred in all samples.

\subsubsection{DBM with perichondrium}

Cartilage formation. Three and seven days after implantation, no cartilage formation was present in the DBM. The perichondrium stained positive for (pro)collagen type I and chondroitin sulfate. In addition, the inner layer of the perichondrium stained positive for collagen type II. After 7 days, cells and loose fibrous tissue was found between the trabeculae of the DBM. Many of the cells stained positive for chondroitin sulfate and occasionally for (pro)collagen type I. After 2 weeks, islets of cartilage were found in the pores of the DBM (Fig. 2). These islets showed positive staining for Alcian Blue, chondroitin sulfate and collagen type II. After 3 weeks the islets of cartilage had fused to a massive cartilaginous structure (Fig. 3). Occasionally, cysts and bloodvessels were found in the cartilage.

Resorption of DBM. After 3 days the perichondrium was markedly thickened. Macrophages were present in the perichondrium tissue and some had also invaded the graft and were attached to DBM. The first 2 weeks after implantation, the number of macrophages in perichondrium as well as DBM gradually increased (Fig. 4). After 2 weeks, the DBM was fragmented and after 3 weeks no signs of DBM were observed any more and the amount of macrophages was strongly reduced. Some macrophages were still present where islets of cartilage were not completely fused. 


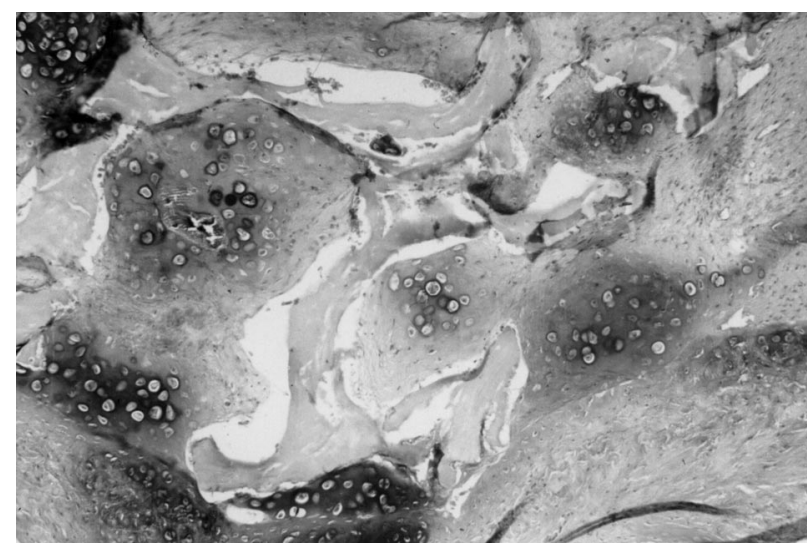

Fig. 2. Cartilage formation in vivo in a composite graft of demineralized bovine bone matrix and ear perichondrium after 2 weeks. Alcian Blue staining, original magnification $100 \times$.

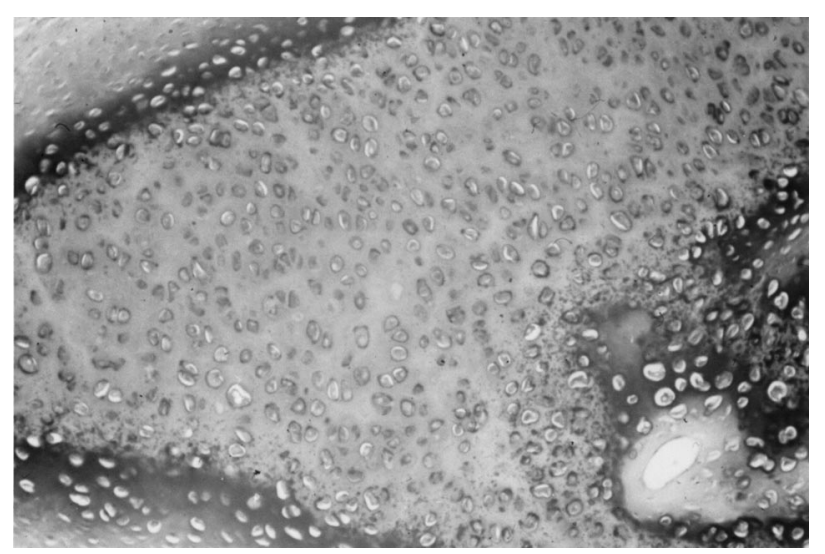

Fig. 3. Cartilage formation in a composite graft of demineralized bovine bone matrix and ear perichondrium, 3 weeks after implantation in vivo. Alcian Blue staining, original magnification $100 \times$.

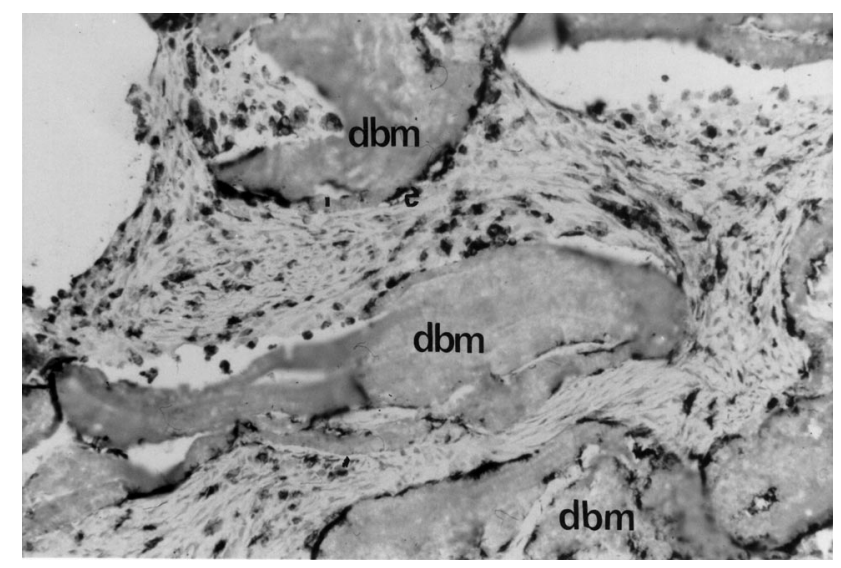

Fig. 4. Macrophages invading the DBM after 14 days implantation in the rabbit ear. Macrophages are stained immunohistochemically with $\mathrm{mAb}$ CD68, sections are counterstained with haematoxylin. Note macrophages are located between the trabeculae of the DBM but also attached to the DBM. Original magnification $200 \times$.
Only a few neutrophils could be detected during the whole period.

\subsection{In vitro}

Both the incidence and the amount of cartilage formed in cultures with 10\% FCS (controls) of auricular perichondrium appeared to be variable. Cartilage formation varied between none or a few chondrocytes to a maximum of half of the explant area (Fig. 5). To investigate the role of DBM in chondrogenesis in vivo, the presence of growth factors in demineralized bovine trabecular bone matrix was tested. Immunohistochemistry demonstrated a faint staining for IGF1 and TGF $\beta 2$, 3 . The absolute amounts could not be determined this way, but staining was generally much lower than in human trabecular bone which had been demineralized by acetic acid and was used as a positive control.

Addition of the recombinant growth factors IGF1, $\mathrm{TGF} \beta 2$ or OP1 to perichondrium cultures in the presence of $10 \%$ FCS, did not improve incidence nor amount of cartilage formation (Table 2). Since we could not demonstrate a difference between continuous or pulsed addition of growth factors, these two conditions were combined in the results. Serum free cultures to which a combination of IGF1 and TGF $\beta 2$ was added for three weeks did not result in stimulation of chondrogenesis.

The use of DBM in perichondrium cultures did not change the incidence nor the amount of cartilage formation after 3 weeks (Table 2). Besides induction of cartilage in the explants, colonization of the DBM by cells was observed (Fig 6). These cells had a round shape and a halo of matrix that stained positively with Alcian Blue. Immunohistochemistry for collagen type II was not feasible on these sections because the DBM did not remain attached to the slide surface during the staining procedure. The variability in the results with DBM were thought to be partly due to variable adhesion of perichondrium tissue to DBM. Attempts were made to advance the adhesion by soaking the DBM in autologous blood before it was wrapped in the perichondrium. This, however, did not influence incidence nor amount of cartilage formation.

In vivo studies have demonstrated that resorption of DBM is performed mainly by macrophages. In vitro macrophages seeded in DBM did not result in visible resorption of the DBM in 3 weeks. Also it did not increase cartilage formation. Combination of blood and macrophages could not mimic the in vivo situation. Since adhesion and activity of the macrophages was questionable, DBM was implanted subcutaneously in the ear for 6 days before culturing with or without perichondrium in vitro. As described in the in vivo experiments, after 6 days subcutaneous implantation, macrophages, mesenchymal cells and some fibrous tissue were present in the DBM but at this point cartilage formation was still 

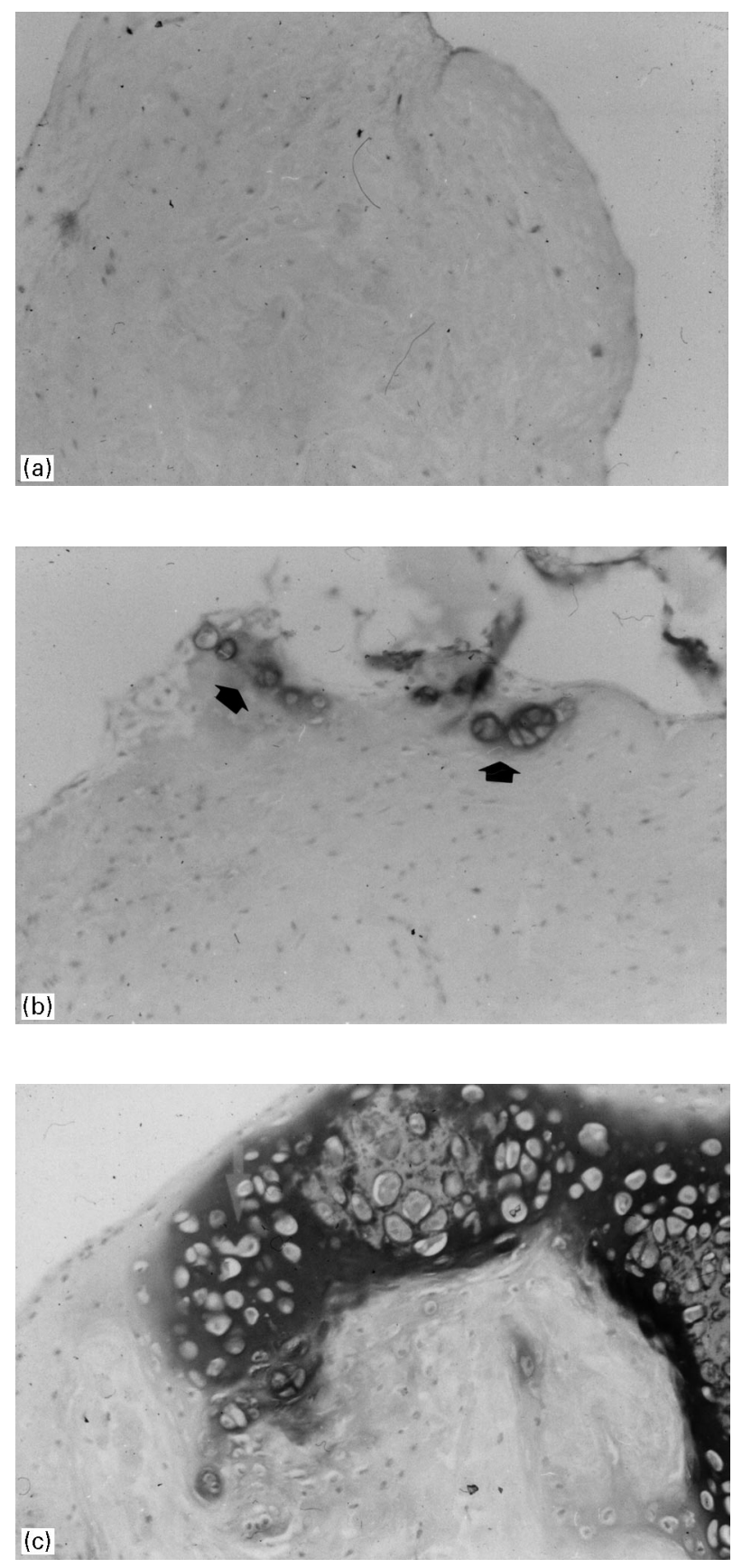

Fig. 5. Chondrogenesis in perichondrium in vitro after 3 weeks, showing the variation in results: (a) no chondrogenesis; (b) a few chondrogenic cells (indicated with arrows); (c) more extensive cartilage formation. Original magnification $200 \times$.

absent. Then this DBM was cultured for 3 weeks with or without a perichondrial envelope, and cartilage formation could be observed in the DBM, however, it was independent of the presence or absence of a perichondrial envelope. Visible resorption of DBM after 6 days in vivo implantation was not observed after 3 weeks of culture.
Table 2

Incidence of chondrogenesis and amount of cartilage formation in vitro in perichondrium after 3 weeks of culture under various conditions. The amount of cartilage formation was quantified using image analysis in the samples where chondrogenesis was present and is presented as \% of the total area of the explant. No statistically significant differences were found

\begin{tabular}{lccl}
\hline \multirow{2}{*}{ Experimental condition } & Incidence & \multicolumn{2}{c}{$\%$ Area cartilage } \\
\cline { 3 - 4 } & & Median & Range \\
\hline Control (10\% FCS) & $16 / 66$ & 9 & $0-32$ \\
TGF- $\beta 2$ & $4 / 17$ & 23 & $6-53$ \\
OP1 & $2 / 16$ & 6 & $0-11$ \\
IGF1 & $1 / 15$ & 2 & n.a. \\
Serum free with IGF1 and TGF $\beta 2$ & $0 / 15$ & n.a. & n.a. \\
DBM & $13 / 47$ & 6 & $1-40$ \\
DBM + macrophages & $1 / 10$ & 3 & n.a. \\
DBM + blood & $6 / 14$ & 10 & $1-47$ \\
DBM + macrophages + blood & $2 / 10$ & 39 & $15-62$ \\
DBM colonized in vivo & $2 / 5$ & 4 & $1-6$ \\
DBM (colonized in vivo) without & & & \\
perichondrium & $1 / 5$ & n.a. & n.a. \\
\hline
\end{tabular}

n.a.: not available.

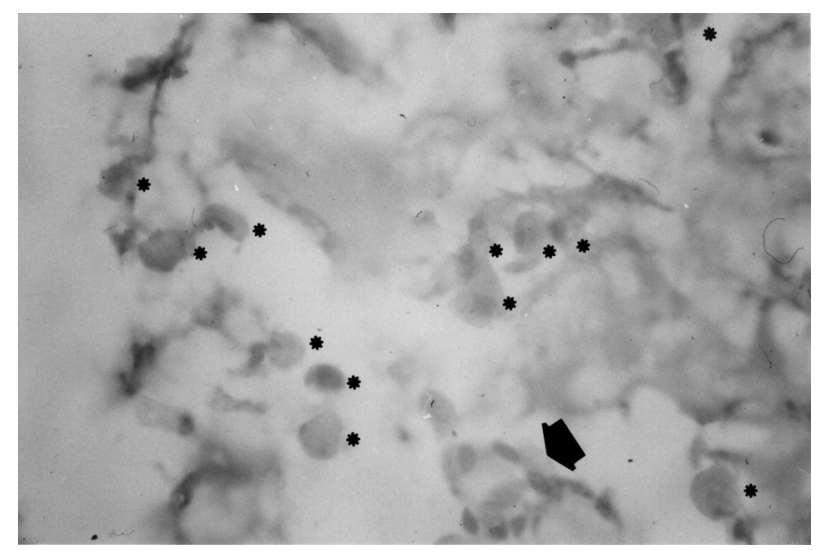

Fig. 6. Cells colonizing the DBM in vitro after 3 weeks. Note the round cell shape and the Alcian Blue staining pericellular matrix. The asterisks indicate the chondrogenic cells, the thick arrow indicate a group of fibroblast-like cells. Original magnification $400 \times$.

\section{Discussion}

In the literature cortical DBM has been demonstrated to contain growth factors which are held responsible for the chondro- and osteogenic potential of this matrix $[9,10]$. The trabecular bovine DBM we used in this study, showed only a faint staining for growth factors. Although in earlier studies addition of growth factors in vitro was demonstrated to induce chondrogenesis in periost [11-16], we could not confirm this for perichondrium; the addition of IGF1, TGF $\beta 2$ or OP1 in vitro did 
not improve chondrogenesis. The difference between perichondrium and periost or the variability in composition of the serum, might explain this discrepancy in results. Yaeger et al. [17] showed that addition of IGF-1 and TGF- $\beta 2$, to cultures in serum-free medium could induce redifferentiation of human articular chondrocytes which had lost their phenotype by culturing in monolayer. Even this combination of IGF-1 and TGF- $\beta 2$ however, did not stimulate cartilage formation from rabbit ear perichondrium.

So this study does not provide evidence for a role of growth factors from the DBM for chondrogenesis. The physical properties of trabecular DBM may be of more importance. Although the present study showed that neither the incidence nor the amount of cartilage generated in vitro was stimulated by the use of DBM, colonization of the DBM by cells could be observed. These cells survived in culture and even had a round shape and produced an Alcian Blue positive matrix, indicating viable cartilage-like cells. This suggests that DBM acts more as a scavenger for chondrogenic cells.

In vivo, resorption of DBM seems to be performed mainly by macrophages. Peritoneal macrophages have been demonstrated to manifest bone resorption [18]. Murine peritoneal macrophages in culture could absorb $40-50 \%$ of small bone particles in two days. In the present study, peritoneal macrophages were isolated, seeded in DBM and cultured together with perichondrium in vitro. Resorption of DBM was not seen macroscopically and contrary to the in vivo situation, DBM was still present after 3 weeks culture. Holtrop et al. [19] suggested that interaction of two cell types is needed for the resorption of bone fragments: one cell type inducing pre-digestion of the matrix with enzymes, and subsequently the macrophages will be able to phagocytize the debris. So probably, macrophages alone are not able to digest such large parts of DBM and addition of enzymes in vitro might be needed to optimize the action of macrophages. Landesman and Reddi [20] reported that the chondro-osteogenic potential of DBM is initially enhanced after implantation in vivo and at its highest point the first 5-7 days in vivo. Therefore, we implanted DBM in vivo for 6 days to obtain colonization with cells and a possible pre-digestion of the DBM, before culturing with perichondrium in vitro for 3 weeks. This combined in vivo/in vitro procedure, however, did not result in visible better resorption of the DBM nor did it increase chondrogenesis.

As another important factor in the in vivo process, the presence of clotted blood in contact with perichondrium is described to be necessary for cartilage formation [21]. Clotted blood consists of many platelets which contain growth factors like PDGF and TGF $\beta$. Cells of the perichondrium are described to grow in the blood clot and form cartilage [22]. However, neither addition of autologous blood nor addition of blood in combination with peritoneal macrophages to our cultures resulted in any stimulation of chondrogenesis.

DBM by itself (without perichondrium) demonstrated no cartilage formation after 3-6 weeks of subcutaneous implantation in vivo. However, culturing in vitro after 6 days of subcutaneous implantation in vivo, chondrogenesis was found in one out of five samples. This indicates that cartilage generation in DBM without perichondrium in principle is possible. Chondrogenic cells were scavenged by the DBM and these cells could originate from the skin $[23,24]$. Subcutaneous implantation of DBM without perichondrium for longer periods (3-6 weeks) never generated cartilage. The reason might be the early resorption of the matrix before cartilage is formed. It could be concluded that because of the fast resorption, pure trabecular bovine DBM is insufficient for cartilage production. Perichondrium is a prerequisite for fast delivery of large amounts of chondrogenic cells. Other matrices have been described in combination with perichondrium [25-27]. When a matrix of collagen, hydroxyapatite or polyglycolic acid was used, increased cartilage formation could be found, however, cartilage did not or minimally invade the biomaterial pores, but mainly lined the biomaterial [25-27]. This again suggests that fast resorption of DBM compared to these other materials might be an important factor in the process of cartilage graft formation.

In vivo, the use of a composite graft of perichondrium and DBM has shown to be a reliable method to generate new cartilage $[6,7]$. Such cartilage formation could not be mimicked in vitro by addition of IGF1, TGF $\beta 2$. OP1, DBM, macrophages or blood. It is suggested that this trabecular DBM has few chondrogenesis inducing qualities but functions mostly as a spacer for cell ingrowth The generation of new cartilage by the proper cells migrating into the 'spacer' together with an optimal period for resorption of the biomaterial makes DBM an excellent matrix for chondrogenesis in vivo.

\section{Acknowledgements}

The authors would like to thank the Department of Pathology of the Erasmus University Rotterdam for the kind hospitality at the laboratory. Pieter Derkx is especially acknowledged for performing the immunohistochemical stainings on DBM. Wim van Vianen of the Department of Clinical Microbiology is acknowledged for his help with the isolation of peritoneal macrophages and the Central Animal Laboratory for taking care of the rabbits.

OP1 was a kind gift of Creative Biomolecules (Dr. K. Sampath). The monoclonal antibody II-II6B3 was obtained from the Developmental Studies Hybridoma Bank maintained by the Department of Pharmacology and Molecular Sciences, John Hopkins University 
School of Medicine, Baltimore, MD, and the Department of Biological Sciences, University of Iowa, Iowa City, IA, under contract N01-HD-6-2915 from the NICHD.

This study was supported by the Sophia Foundation and by the Technology Foundation (STW), applied science division of NWO and the technology programme of the Ministry of Economic Affairs.

\section{References}

[1] Skoog T, Ohlsen L, Sohn SA. Perichondrial potential for cartilage regeneration. Scand J Plast Reconstr Surg 1972;6:123-5.

[2] Engkvist $O$, Wilander E. Formation of cartilage from rib perichondrium grafted to an articular defect in the femur condyle of the rabbit. Scand J Plast Reconstr Surg 1979;13:371-6.

[3] Engkvist O, Skoog V, Pastacaldi P, Yormuk E, Juhlin R. The cartilaginous potential of the perichondrium in rabbit ear and rib. A comparative study in vivo and in vitro. Scand J Plast Reconstr Surg 1979;13:269-74.

[4] Homminga GN, Van der Linden TJ, Terwindt-Rouwenhorst EAW, Drukker J. Repair of articular defects by perichondrial grafts. Experiments in the rabbit. Acta Orthop Scand 1989;60:326-9.

[5] Homminga GN, Bulstra SK, Bouwmeester PS, Van der Linden AJ. Perichondral grafting for cartilage lesions of the knee. J Bone Jt Surg 1990;72B:1003-7.

[6] Bean JK, Verwoerd-Verhoef HL, Verwoerd CDA, van der Heul RO. Chondrogenesis in a collagen matrix. In: Dixon A, Sarnat B, editors. Fundamentals of bone growth. Boca Raton, USA: CRC Press, 1991:113-20.

[7] Ten Koppel PGJ, van Osch GJVM, Verwoerd-Verhoef HL. A method to generate a cartilage graft; perichondrium combined with spongeous demineralized bovine bone matrix. Trans ORS 1997;22:538.

[8] Pirsig W, Bean JK, Lenders H, Verwoerd CDA, Verwoerd-Verhoef HL. Cartilage transformation in a composite graft of demineralized bovine bone matrix and ear perichondrium used in a child for the reconstruction of the nasal septum. Int J Ped ORL 1995;32:171-81.

[9] Urist MR, Mikulski A, Lietze A. Solubilized and insolubilized bone morphogenetic protein. Proc Natl Acad Sci USA 1979;75:1828-32.

[10] Sampath TK, Nathanson MA, Reddi AH. In vitro transformation of mesenchymal cells derived from embryonic muscle into cartilage in response to extracellular matrix components of bone. Proc Natl Acad Sci USA 1984;81:3419-23.

[11] Dieudonné SC, Semeins CM, Goei SW, Vukicevic S, Klein Nulend J, Sampath TK, Helder M, Burger EH. Opposite effects of osteogenic protein and tranforming growth factor $\beta$ on chondrogenesis in cultured long bone rudiments. J Bone Miner Res 1994:9:771-80.
[12] Iwasaki M, Nakata K, Nakahara H, Nakase T, Kimura T, Kimata K, Caplan AI, Ono K. Transforming growth factor- $\beta 1$ stimulates chondrogenesis and inhibits osteogenesis in high density culture of periosteum-derived cells. Endocrinology 1993; 132:1603-8.

[13] Izumi T, Scully SP, Heydemann A, Bolander ME. Transforming growth factor $\beta 1$ stimulates type II collagen experession in cultured periosteum-derived cells. J Bone Miner Res 1992; 7:115-21

[14] Moar G, Hochberg Z, Silbermann M. Insulin-like growth factor I accelerates proliferation and differentiation of cartilage progenitor cells in cultures of neonatal mandibular condyles. Acta Endocrinol 1993;128:56-64.

[15] Miura Y, Fitzsimmons JS, Commisso CN, Gallay SH, O'Driscoll SW. Enhancement of periosteal chondrogenesis in vitro. Doseresponse for transforming growth factor- $\beta 1$. Clin Orthop Relat Res 1994;301:271-80.

[16] O’Driscoll SW, Recklies AD, Poole AR. Chondrogenesis in periosteal explants. An organ culture model for in vitro studies. J Bone Jt Surg 1994;76a:1042-51.

[17] Yaeger P, Kaluzhny J, Masi TL, Tubo R, McPherson J, Binette F. Synergistic action of TGF- $\beta$ and IGF-1 is sufficient for redifferentiation of adult human articular chondrocytes in defined medium. Trans Orthop Res Soc 1997;22:515.

[18] Teitelbaum SL, Stewart CC, Kahn AJ. Rodent peritoneal macrophages as bone resorbing cells. Calcif Tissue Int 1979;27:255-61.

[19] Holtrop M, Cox KA, Glowacki J. Cells of the mononuclear phagocytic system resorb implanted bone matrix: a histologic and ultrastructural study. Calcif Tissue Int 1982;34:488-94.

[20] Landesman R, Reddi AH. In vivo analysis of the half-life of the osteoinductive potential of demineralized bone matrix using diffusion chambers. Calcif Tissue Int 1989;45:348-53.

[21] Donski P, O’Brien BMcC. Perichondrial microvascular free transfer: an experimental study in rabbits. Br J Plast Surg 1980;33:46-53.

[22] Ohlsen L. Cartilage regeneration from perichondrium. Experimental studies and clinical applications. Plast Reconstr Surg 1978;62:507-13.

[23] Inoue T, Deporter DA, Melcher AH. Induction of chondrogenesis in muscle, skin, bone marrow and periodontal ligament by demineralized dentin and bone matrix in vivo and in vitro. J Dent Res 1986;65:12-22.

[24] Glowacki J. Cellular reactions to bone-derived material. Clin Orthop Relat Res 1996;324:47-54.

[25] Verwoerd CD, Adriaansen FC, van der Heul RO, VerwoerdVerhoef HL. Porous hydroxylapatite-perichondrium graft in cricoid reconstruction. Acta Oto-Laryngol 1987;103:496-502.

[26] Ruuskanen MM, Virtanen MK, Tuominen H, Tormala P, Waris T. Generation of cartilage from auricular and rib free perichondrial grafts around a self-reinforced polyglycolic acid mould in rabbits. Scand J Plast Reconstr Surg Hand Surg 1994;28:81-6.

[27] Matsuda K, Nagasawa N, Suzuki S, Isshiki N, Ikada Y. In vivo chondrogenesis in collagen sponge sandwiched by perichondrium. J Biomater Sci Polym Ed 1995;7:221-9. 